\title{
openheart Postdischarge outcome after Takotsubo syndrome compared with patients post- ACS and those without prior CVD: ANZACS-QI 19
}

Jen-Li Looi, ${ }^{1}$ Mildred Lee, ${ }^{1}$ Mark W I Webster, ${ }^{2}$ Andrew C Y To, ${ }^{3}$ Andrew J Kerr ${ }^{1}$

To cite: Looi J-L, Lee M, Webster MWI, et al. Postdischarge outcome after Takotsubo syndrome compared with patients post-ACS and those without prior CVD: ANZACS-QI 19. Open Heart 2018;5:e000918. doi:10.1136/ openhrt-2018-000918

Received 11 August 2018 Revised 25 September 2018 Accepted 12 November 2018

Check for updates

(c) Author(s) (or their employer(s)) 2018. Re-use permitted under CC BY-NC. No commercial re-use. See rights and permissions. Published by BMJ.

${ }^{1}$ Department of Cardiology, Middlemore Hospital, Auckland, New Zealand

${ }^{2}$ Green Lane Cardiovascular Service, Auckland City Hospital, Auckland, New Zealand

${ }^{3}$ Cardiovascular Division, North Shore Hospital, Takapuna, Auckland, New Zealand

Correspondence to Dr Jen-Li Looi; JenLi.Looi@ middlemore.co.nz

\section{ABSTRACT}

Objective Takotsubo syndrome (TS) mimics acute coronary syndrome (ACS) but has a distinct pathophysiology. While in-hospital adverse outcomes appear similar to those presenting with an ACS, data on longer term postdischarge risk are conflicting. This study sought to assess the long-term prognosis of patients discharged alive after TS.

Methods The clinical profile and in-hospital and long-term outcomes were prospectively assessed in consecutive patients with TS. Survival in patients with TS was compared with two representative age-matched and gender-matched comparison cohorts: a hospitalised ACS cohort and a community cohort without known cardiovascular disease (CVD).

Results Two hundred and-twenty-five patients with TS (216 women, mean age $63.7 \pm 11.8$ years) were included. In-hospital mortality was $1.8 \%$ and $1.9 \%$ for patients with TS and ACS, respectively. Of the 219 patients with TS with postdischarge follow-up, at a mean follow-up of $4.8 \pm 3.2$ years, there were $19(8.3 \%)$ deaths, 18 of which were from non-cardiac causes. When compared with the cohort without prior CVD, postdischarge patients with TS were at increased mortality risk (HR 2.00, 95\% Cl 1.26 to 3.17 , $\mathrm{p}=0.003$ ), but mortality in postdischarge patients with ACS was over threefold higher (HR 3.43, 95\% Cl 2.97 to 3.96, $\mathrm{p}<0.0001$ ).

Conclusions In-hospital mortality for patients diagnosed with TS and ACS was similar. However, while postdischarge survivors of TS had a long-term survival which was poorer than for a community-based cohort without known CVD, their survival was better than for postdischarge survivors of an ACS event. Late deaths in patients with TS were almost all from non-cardiac causes.

\section{INTRODUCTION}

Takotsubo syndrome (TS) (also known as apical ballooning syndrome or stress-induced transient left ventricular [LV] dysfunction) is characterised by acute but rapidly reversible LV dysfunction with distinct wall motion abnormalities, predominantly affecting postmenopausal women. ${ }^{1-3}$

The initial presentation of TS mimics acute coronary syndrome (ACS), and the

\section{Key questions}

What is already known about this subject?

- Takotsubo syndrome (TS) (also known as apical ballooning syndrome or stress-induced transient left ventricular [LV] dysfunction) is characterised by acute but rapidly reversible LV dysfunction with distinct wall motion abnormalities, predominantly affecting postmenopausal women after a stressful event.

- There are conflicting reports regarding the longterm prognosis in patients with TS who survive the acute event.

What does this study add?

- We studied patients with TS with two representative age-matched and gender-matched comparison cohorts: a hospitalised acute coronary syndrome (ACS) cohort and a community cohort without known cardiovascular disease (CVD).

- Our study demonstrated the in-hospital mortality for patients diagnosed with TS and ACS was virtually identical.

- However, while postdischarge survivors of TS had a long-term survival which was poorer than for a community-based cohort without known CVD, their survival was better than for postdischarge survivors of an ACS event.

- In addition, late deaths in the TS cohort were almost all from non-cardiac causes and appear to be related to the presence of comorbid disease rather than the TS event itself.

How might this impact on clinical practice?

- Patients with TS who recover from the initial event can be reassured that their risk of future cardiovascular death is no higher than that for the general population without CVD.

- They do have an approximately 1 in 20 risk of recurrent TS in the next 5 years, and they are at a slightly higher absolute risk of dying in the longer term from a non-cardiac cause compared with the general population without CVD.

acute impairment of LV systolic function can lead to serious complications, including cardiogenic shock, congestive heart failure 
and lethal arrhythmias, with an in-hospital mortality of $2.0 \%-8.7 \%,{ }^{4-14}$ a mortality rate similar to that seen in ACS. ${ }^{3}$ The underlying pathophysiology of TS is, however, distinct from ACS, and there is usually complete recovery of LV function within several weeks. ${ }^{15}$ Recurrent TS is well recognised, but is relatively infrequent and usually non-fatal. ${ }^{1314}$ In contrast the progression of atherosclerosis and irreversible myocardial damage characteristic of ACS is associated with an increased risk of adverse longterm outcomes. ${ }^{16-18}$ The difference in underlying pathophysiology suggests that longer term outcomes in TS may differ from those after an ACS.

There are conflicting reports regarding the long-term prognosis in patients with TS who survive the index event. ${ }^{3} 513$ 19-22 When longer term outcomes of patients with TS were compared with those in $\mathrm{ACS}^{21-23}$ and non-cardiovasculardisease (CVD) control cohorts, findings ranged from all-cause mortality in TS being similar to the general population, ${ }^{13}$ to TS outcomes as bad as for patients with ST-segment elevation myocardial infarction (STEMI) ${ }^{21}$ The conflicting observations may in part relate to methodological limitations, including not separating early in-hospital from postdischarge outcomes, and not using representative 'control' cohorts. Non-CVD control groups were drawn from a cohort of patients with normal coronary arteries by angiography in one study ${ }^{22}$ and a STEMI control group from a clinical trial population in another. ${ }^{23}$

In Auckland, New Zealand, all patients diagnosed with TS in each of the three major hospitals admitting acute cardiac patients are prospectively collected in a registry. ${ }^{14}$ New Zealand also has comprehensive national hospital discharge coding and community CVD risk assessment data sets which allow us to compare outcomes in patients with TS with large contemporaneous ACS and community 'no known CVD' cohorts. This study aims to describe the postdischarge outcome of patients with TS, compared with age-matched and gender-matched ACS and 'no known CVD' control groups.

\section{METHODS}

\section{Patient population}

Patients presenting with TS were prospectively identified from the three major public hospitals in Auckland (Middlemore Hospital, Auckland City Hospital and North Shore Hospital), New Zealand, between March 2004 and October 2015. These hospitals receive all patients with ACS and TS hospitalised in the northern region of New Zealand, representing a third of ACS admissions. Only patients who underwent cardiac angiography (CT or invasive) to confirm they met the diagnostic criteria for TS were included in the study. TS was defined using the criteria proposed by the InterTAK Diagnostic Criteria $^{24}$ : (1) transient LV dysfunction (hypokinesia, akinesia or dyskinesia) presenting as apical ballooning or mid-ventricular, basal or focal wall motion abnormalities; regional wall motion abnormality usually extends beyond a single epicardial vascular distribution; however, rare cases can exist where the regional wall motion abnormality is present in the subtended myocardial territory of a single coronary artery (focal TS); right ventricular involvement can be present; (2) preceding emotional, physical or combined trigger, but this is not obligatory; (3) neurological disorders (eg, subarachnoid haemorrhage, stroke/transient ischaemic attack or seizures) as well as pheochromocytoma may serve as triggers; (4) presence of new ECG abnormalities (ST-segment elevation, ST-segment depression, T-wave inversion and QTc prolongation); however, rare cases exist without any ECG changes; (5) moderate elevation in cardiac biomarkers (troponin and creatine kinase) in most cases; significant elevation of brain natriuretic peptide is common; (6) significant coronary artery disease $(\mathrm{CAD})$ is not a contradiction; (7) absence of infectious myocarditis; and (8) postmenopausal women are predominantly affected.

Clinical, ECG, echocardiographic and angiographic data of the study population were obtained as previously described. ${ }^{14}$ Late follow-up data were obtained by reviewing the medical records of each patient or telephone interview with patients' general practitioners. We recorded in-hospital complications, including acute pulmonary oedema, cardiogenic shock, use of invasive or non-invasive ventilation or intra-aortic balloon pump (IABP) insertion, and death.

The primary outcome was mortality in those patients discharged alive. We also report rehospitalisation for recurrence of TS, which was defined using the InterTAK Diagnostic Criteria, except that in most cases coronary angiography was not repeated.

\section{Survival analysis}

Survival of patients with TS from hospital discharge was compared with age-matched and gender-matched cohorts of patients with ACS and people without known CVD.

\section{ACS cohort}

National hospital data on all ACS admissions (primary or secondary International Classification of Diseases 10th Revision codes 120.0, 121 and 122) to participating hospital catchments were obtained for the period between 1 January 2005 and 30 December 2013. Only patients who had coronary angiography were included to ensure comparability with the TS cohort. An age-matched and gender-matched ACS comparison cohort was identified by matching to TS cases by sex, age group $(<50,50-60$, $60-70,70-80$ and $80+$ years) and admission period. Using these matching parameters, 13 patients with ACS were able to be identified for each patient with TS.

\section{Cohort without known CVD}

This cohort were people who received a routine CVD risk assessment in primary care practices which use the PREDICT decision support software. The PREDICT cohort comprised 218052 individuals, representing 
approximately half of those eligible for routine CVD risk assessment in the region. ${ }^{25}$ Patients were clinically stable and living in the community at the time of registration.

An age-matched and gender-matched 'no known CVD' comparison cohort was obtained using patients who had undergone their first PREDICT-CVD risk assessment between 2 March 2004 and 24 July 2012. The cohort was matched to TS cases by sex and age group (as above) and date of assessment. Using these matching parameters 100 patients without prior CVD were able to be identified for each patient with TS.

Exclusion criteria for the cohort without known CVD were history of CVD, angina medication and a high CVD risk condition (including atrial fibrillation, diabetes with confirmed microalbuminuria or overt nephropathy, estimated glomerular filtration rate $\leq 30 \mathrm{~mL} / \mathrm{min} / 1.73$ $\mathrm{m}^{2}$, and those dispensed loop diuretics, as a proxy for congestive heart failure). ${ }^{26}$ These additional CVD risk factors were obtained from the PREDICT risk assessment or through linkage to national hospitalisation and drug dispensing databases, and a regional laboratory results database.

\section{Statistical analysis}

Categorical data were summarised as frequency and percentages. Continuous data were presented as mean $\pm \mathrm{SD}$ and/or median (IQR). Kaplan-Meier survival curves were constructed from the date of discharge (TS and ACS cohorts) and date of CVD risk assessment (no known CVD cohort) and compared using bivariate log-rank tests. Univariate Cox models were used to quantify the risk of postdischarge mortality in each of the TS and ACS groups compared with those without known CVD. Data were analysed using SAS V.9.4 statistical package.

\section{RESULTS}

\section{Study population}

Two hundred and twenty-five consecutive patients with TS were included in the study. The clinical characteristics of patients with TS are presented in table 1 . The mean age at presentation was $63.7 \pm 11.8$ years (median 66 years) and $216(96 \%)$ were women. The ACS $(n=2925)$ and no prior CVD ( $n=22500)$ groups were well matched with $96 \%$ women in each and mean age and SD of $64 \pm 12$ years. The most common presenting symptoms in patients with TS were chest pain and dyspnoea. Twenty-seven patients presented with radiological evidence of pulmonary oedema and 12 with a ventricular arrhythmia ( 7 with ventricular fibrillation and 5 with ventricular tachycardia). Sixty-eight patients (29.6\%) had ST-segment elevation on their admission ECG. A stressful trigger (defined as an unusual emotional or physical stress occurring before symptom onset) was identified in 165 patients. Emotional triggers were more frequent than physical triggers (44\% and 29\%, respectively). The physical precipitating factors for TS are listed in table 2. In $27 \%$ of patients, TS occurred without an obvious trigger.
Table 1 Characteristics of patients with Takotsubo syndrome

\begin{tabular}{|c|c|}
\hline & $\begin{array}{l}\text { Total cohort } \\
\mathrm{N}=225\end{array}$ \\
\hline Female & $216(96.0)$ \\
\hline Age (years), mean $\pm S D$ & $64 \pm 11.8$ \\
\hline \multicolumn{2}{|l|}{ Ethnicity } \\
\hline European & $165(73.3)$ \\
\hline New Zealand Maori & $36(16.0)$ \\
\hline Pacific Islanders & $13(5.8)$ \\
\hline Asian & $11(4.9)$ \\
\hline Hypertension & $85(37.8)$ \\
\hline Dyslipidaemia & $64(28.4)$ \\
\hline Diabetes & $21(9.3)$ \\
\hline Current smoker & $32(14.2)$ \\
\hline Stressor on admission & $165(73.3)$ \\
\hline \multicolumn{2}{|l|}{ Type of stressor } \\
\hline Emotional & $99(44)$ \\
\hline Physical & $66(29)$ \\
\hline None identified & $60(27)$ \\
\hline \multicolumn{2}{|l|}{ Symptoms on admission } \\
\hline Chest pain & $181(80.4)$ \\
\hline Dyspnoea & $63(28.0)$ \\
\hline \multicolumn{2}{|l|}{ Arrhythmia on admission } \\
\hline Atrial arrhythmia & $11(4.9)$ \\
\hline Ventricular arrhythmia & $12(5.3)$ \\
\hline Pulmonary oedema on chest X-ray & $27(12.0)$ \\
\hline IABP insertion & $6(2.7)$ \\
\hline Intubation & $9(4.0)$ \\
\hline CPAP ventilation & $3(1.3)$ \\
\hline ST elevation on admission & $68(30.2)$ \\
\hline \multicolumn{2}{|l|}{ LV systolic dysfunction during acute phase } \\
\hline Normal/Low normal & $50(22.2)$ \\
\hline Mild & $61(27.1)$ \\
\hline Moderate & $80(34.2)$ \\
\hline Severe & $34(14.5)$ \\
\hline Normal coronary anatomy & $133(59.1)$ \\
\hline \multicolumn{2}{|l|}{ In-hospital outcomes } \\
\hline Death & $4(1.8)$ \\
\hline Days of hospitalisation, median (IQR) & $4(3-7)$ \\
\hline
\end{tabular}

Values are $n(\%)$ unless otherwise stated.

CPAP, continuous positive airway pressure; IABP, intra-aortic balloon pump; LV, left ventricle.

More than half of the patients with TS had a significant reduction in LV ejection fraction during the acute phase. All patients underwent either invasive coronary angiography $(n=219)$ or CT coronary angiography $(n=6)$ during the acute admission: 133 patients had normal coronary arteries and 62 had mild disease ( $<50 \%$ stenosis). Threevessel disease was diagnosed in six patients, and these patients were included in the study as regional wall 


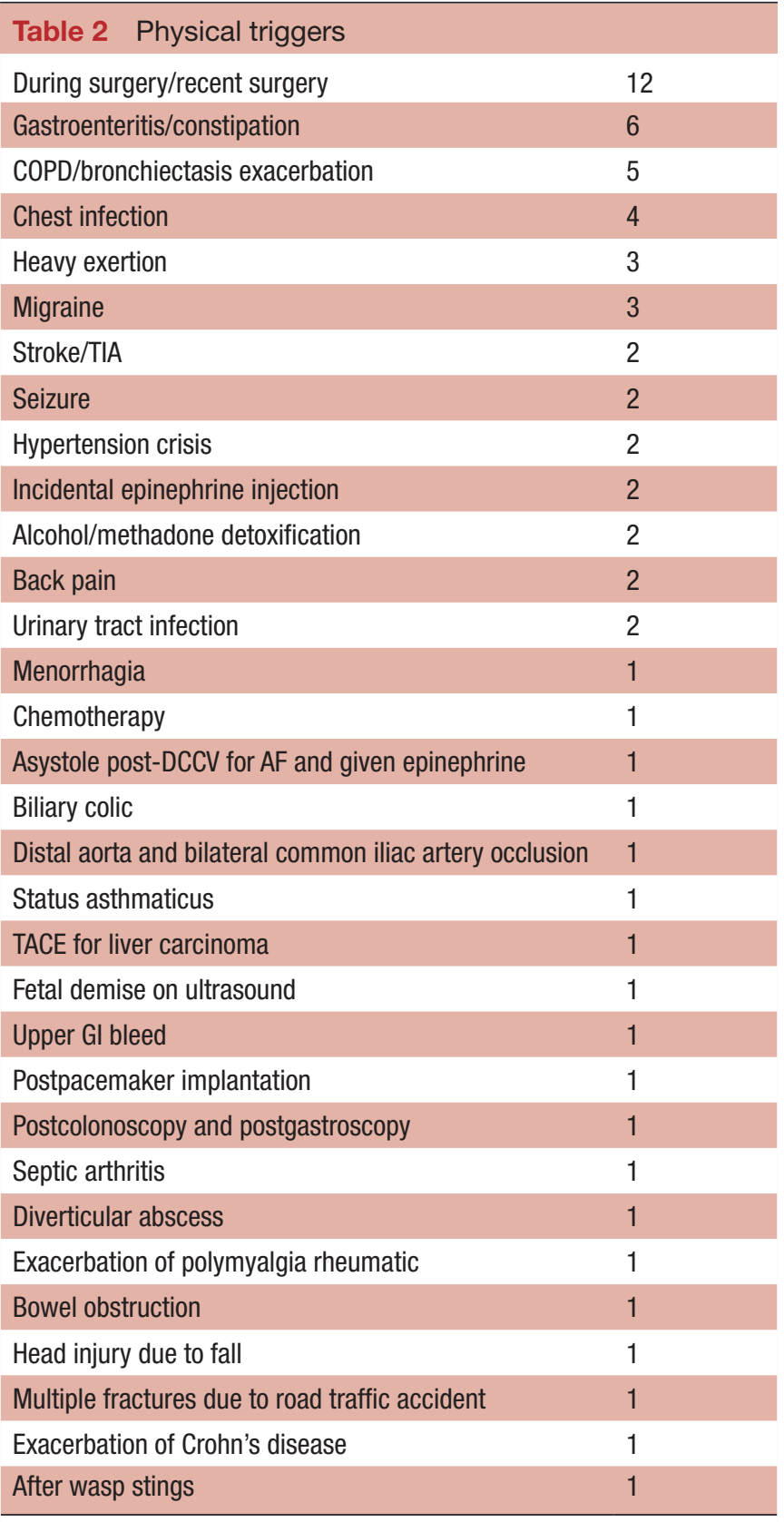

AF, atrial fibrillation; COPD, chronic obstructive pulmonary disease; DCCV, direct current cardioversion; GI, gastrointestinal; TACE, transarterial chemoembolisation; TIA, transient ischaemic attack.

motion abnormalities extended beyond a single-vessel distribution.

\section{In-hospital clinical course}

Four patients with TS $(1.8 \%)$ died during the index admission from cardiogenic shock. In the ACS cohort there were 55 in-hospital deaths $(1.9 \%)$.

In-hospital complications occurred in $30(13 \%)$ of the TS. During the index hospitalisation, nine patients were intubated, of whom six also had IABP insertion. Twenty-seven patients had radiological evidence of pulmonary oedema. Of these 27 patients, 8 were intubated, 4 required IABP insertion and 3 required continuous positive airway pressure ventilation. Two hundred and

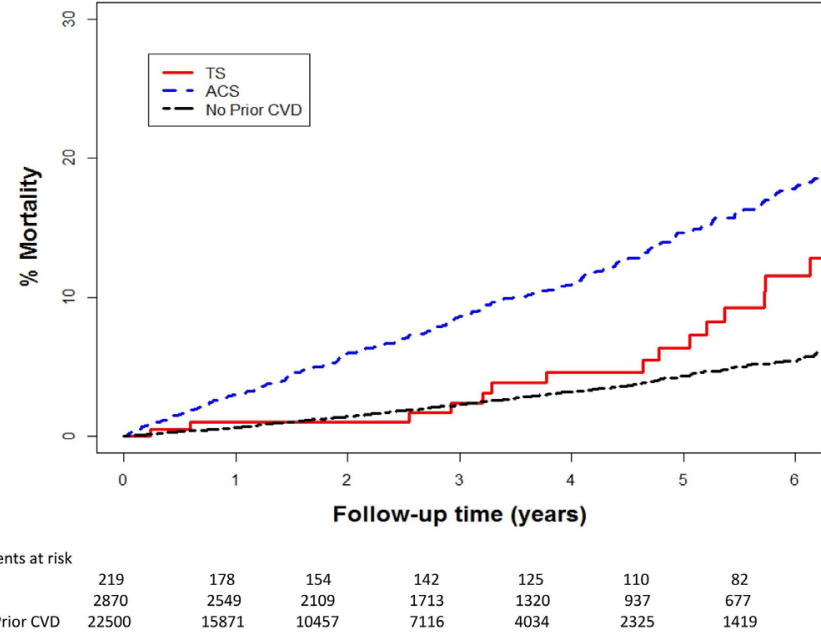

$\begin{array}{lllllll}\text { No Prior CVD } & 22500 & 15871 & 10457 & 7116 & 4034 & 2325\end{array}$

Figure 1 Cumulative mortality of patients with TS versus age-matched and gender-matched population with ACS and with no known CVD. Log-rank test: TS versus ACS, $p<0.0001$; TS versus no known CVD, $p<0.0001$; ACS versus no known CVD, $p<0.0001$. ACS, acute coronary syndrome; CVD, cardiovascular disease; TS, Takotsubo syndrome.

twenty-one patients with TS and 2870 patients with ACS were discharged alive after the index hospitalisation.

\section{Postdischarge outcomes}

The mean follow-up for the TS cohort was $4.8 \pm 3.2$ years (median 5.0 years). Two patients with TS were not included in this analysis because they were non-residents and had no follow-up in New Zealand after discharge. For the ACS and no prior CVD cohorts, the mean follow-up times were $4.0 \pm 2.5$ and $2.3 \pm 1.9$, respectively. Figure 1 shows the mortality of the TS population compared with the ACS and no prior CVD cohorts. Compared with the cohort without CVD, patients with TS postdischarge had about twice the mortality risk (HR 2.00 [95\% CI 1.26 to 3.17]), but those with ACS had an over threefold higher (HR 3.43 [95\% CI 2.97 to 3.96]) mortality risk than the cohort without prior CVD. Inspection of the mortality plots shows that the TS and no known CVD curves only began to diverge after about 4 years. Among women in the TS cohort, 18 of the 19 deaths were from non-cardiac causes: 4 cancer, 4 cerebrovascular events, 4 end-stage chronic airway disease, 2 sepsis, 1 respiratory failure due to severe organising pneumonitis, 1 liver failure due to cirrhosis, 1 significant drop in haemoglobin without source of bleeding identified and 1 trauma. One patient died from end-stage severe right heart failure.

Fourteen $(6.2 \%)$ of 225 patients, all women, experienced recurrent TS, none of which were fatal.

\section{DISCUSSION}

This study describes the natural history of TS in a prospectively enrolled 225 patient cohort with near-complete follow-up to a mean of almost 5 years. The major strength of this study compared with prior studies is that the longterm postdischarge outcomes were able to be compared 
with two very large, real-world, matched cohorts of patients presenting with ACS and people without known CVD, respectively. The postdischarge mortality risk for those with TS was approximately twofold higher than for patients in the community without known CVD, but for patients with ACS it was over threefold higher.

\section{In-hospital outcomes}

In contrast to the superior long-term outcomes in TS survivors compared with patients with ACS, the in-hospital mortality rate was virtually identical (1.8\% vs $1.9 \%)$.

The in-hospital mortality for patients with TS in this study compares favourably with the $2 \%-5 \%$ in-hospital mortality rate in other series. ${ }^{5621}$ In a meta-analysis of 2120 patients with TS in 37 studies, the in-hospital mortality rate was $4.5 \%,{ }^{11}$ and was $4.2 \%$ in the large National Inpatient Sample-USA TS cohort. ${ }^{9}{ }^{27}$ The in-hospital mortality of patients with TS in these studies is comparable with that observed in patients with ACS receiving current state-of-the-art management. ${ }^{321}$

As found in those series, death was mainly from refractory cardiogenic shock or ventricular fibrillation. ${ }^{61}$ In addition, a third of our patients with TS experienced potentially life-threatening complications during the acute phase, related to haemodynamic instability, including $27 \%$ with pulmonary oedema and $5 \%$ with cardiogenic shock. This is similar to the in-hospital complication rates described in other cohorts, ${ }^{5}{ }^{28}$ suggesting that the spectrum of TS is wide and ranges from low to very high risk in the acute phase. The relatively rapid recovery of $\mathrm{LV}$ function and a selection bias towards low-risk patients in previous reports may have generated the misapprehension that this condition is usually benign. ${ }^{24}$

\section{Longer term survival}

\section{TS compared with a 'normal' control population}

There are few studies which have attempted to compare outcomes with a well-matched control group without known CVD. We found that having TS was associated with a postdischarge HR for mortality of 2.00 compared with that of age-matched and gender-matched control patients drawn from a contemporaneous, representative population cohort. The most comparable study is a recent Swedish study ${ }^{22}$ which reported better survival in a control group drawn from a cohort with chest pain of unknown cause and normal coronary arteries. In that study the HR for mortality associated with TS (2.1) was very similar to our finding. However, in that study in-hospital mortality was included in the outcome analysis, precluding comparison of postdischarge outcomes. In addition, it is also possible that their control patients may have been at higher risk than a general population cohort. Two other prior studies ${ }^{513}$ compared longer term outcomes with the Minnesota age-matched and gendermatched general population and reported conflicting results, with one showing no difference in long-term survival and the other reporting, as we did, that patients with TS fared worse. We note that those studies included in-hospital events in their analyses and that the control group would have included those with prior CVD living in the community, which may have increased the event rate in controls.

\section{Patients with TS compared with patients with ACS}

The postdischarge risk of death associated with ACS was over threefold higher than in the cohort without prior CVD, which was higher than the doubling of risk observed in patients with TS. There are a number of studies that have compared longer term outcomes in TS with various ACS cohorts, ${ }^{21-23}$ although to our knowledge only one study $^{23}$ has separately analysed early and postdischarge mortality. There is an early hazard after ACS, with half of the first-year mortality occurring in the first month. ${ }^{29}$ Including these early outcomes with the later ones masks the assessment of whether late outcomes diverge between ACS and TS. Despite this limitation, a number of studies have reported that the long-term prognosis of TS survivors is more benign than after an ACS. ${ }^{12430}$ However more recent studies, in contrast to those earlier studies and the current study, have found comparable ${ }^{321}$ or even worse outcomes in patients with $\mathrm{TS},{ }^{23}$ when compared with patients with ACS. Methodological differences may explain some of the differences between studies. In the study reporting worse outcomes in patients with TS, the STEMI comparison cohort was drawn from a clinical trial cohort, which may be a relatively lower risk cohort than our more comprehensive 'real world' cohort.

\section{Postdischarge deaths}

Of the patients with TS discharged alive in our cohort, $8.4 \%$ died during follow-up, with all but 1 of the 19 deaths from non-cardiac causes. This is consistent with other studies which have reported that late mortality after TS is largely due to non-cardiac causes, ${ }^{513}$ and suggests that late mortality is due to the comorbid disease rather than the TS event itself. The variation in reported late mortality discussed above may be due to differences in the frequency of underlying severe comorbidities between cohorts. In contrast, mortality after ACS is most commonly due to CVD.

\section{TS recurrence}

Six per cent of our surviving patients experienced recurrent and remarkably similar TS episodes. Our findings were similar to those of Sharkey et $a l^{5}{ }^{5}$ who reported a recurrence rate of $5 \%$.

\section{Clinical implications}

Patients in our population with a definite diagnosis of TS who recover from the initial event can be reassured that their risk of future cardiovascular death is no higher than that for the general population without CVD. They do have an approximately 1 in 20 risk of recurrent TS in the next 5 years, and they are at a slightly higher absolute risk of dying in the longer term from a non-cardiac cause compared with their peers without CVD. 


\section{Study limitations}

Despite prospectively capturing all patients presenting with TS in Auckland over 11.5 years, the study population is only of moderate size and our analysis includes a relatively small number of hard endpoints, which limit the conclusions that can be drawn. Although our analyses have controlled for age and gender, we were unable to control for other possible confounders as consistent comorbidity and medication information is not available in the three separate data sets used for this study. In particular, comorbid medical conditions may be more frequent in the TS cohort than in the no prior CVD cohort, which would contribute to the observed excess mortality risk in patients with TS.

Concomitant CAD in TS is reported with a prevalence ranging from $10 \%$ to $29 \% .^{3}$ Thus, the presence of CAD should not be considered as an exclusion criterion for TS and we wanted to be certain as possible about both the ACS and TS diagnoses for this study; we applied these criteria rigorously and only included patients who had angiography.

In this study the cause of death in the ACS cohort was not available. In prior studies ${ }^{321}$ cardiac causes of death have predominated in contrast to what we have described in the TS cohort in the current study.

\section{CONCLUSION}

In this study the in-hospital mortality for patients diagnosed with TS and ACS was virtually identical. However, while postdischarge survivors of TS had a long-term survival which was poorer than for a community-based cohort without known CVD, their survival was better than for postdischarge survivors of an ACS event. In addition, late deaths in the TS cohort were almost all from non-cardiac causes and appear to be related to the presence of comorbid disease rather than the TS event itself.

Contributors J-LL was responsible for data collection and drafting the manuscript. $M L$ was responsible for statistical analysis of the data. MW and ACYT were responsible for data collection and reviewing the manuscript. AJK was responsible for drafting and reviewing the manuscript.

Funding The authors have not declared a specific grant for this research from any funding agency in the public, commercial or not-for-profit sectors.

\section{Competing interests None declared.}

Patient consent for publication Not required.

Ethics approval The TS registry and outcome study was approved by the Health and Disability Ethics Committees as a clinical audit (NTX/11/EXP/288). The ACS and no prior CVD cohorts were available via the VIEW research programme, which was approved by the Northern Y Regional Ethics Committee in 2003 (AKY/03/12/314), with subsequent annual reapproval by the national Multi-Region Ethics Committee since 2007 (MEC/01/19/EXP). Individual patient consent was not required as all data are anonymised.

Provenance and peer review Not commissioned; externally peer reviewed.

Data sharing statement No additional data are available.

Open access This is an open access article distributed in accordance with the Creative Commons Attribution Non Commercial (CC BY-NC 4.0) license, which permits others to distribute, remix, adapt, build upon this work non-commercially, and license their derivative works on different terms, provided the original work is properly cited, appropriate credit is given, any changes made indicated, and the use is non-commercial. See: http://creativecommons.org/licenses/by-nc/4.0

\section{REFERENCES}

1. Parodi G, Del Pace S, Carrabba N, et al. Incidence, clinical findings, and outcome of women with left ventricular apical ballooning syndrome. Am J Cardiol 2007;99:182-5.

2. Bybee KA, Prasad A, Barsness GW, et al. Clinical characteristics and thrombolysis in myocardial infarction frame counts in women with transient left ventricular apical ballooning syndrome. Am J Cardiol 2004;94:343-6.

3. Templin C, Ghadri JR, Diekmann J, et al. Clinical Features and Outcomes of Takotsubo (Stress) Cardiomyopathy. N Engl J Med 2015;373:929-38.

4. Sharkey SW, Lesser JR, Zenovich AG, et al. Acute and reversible cardiomyopathy provoked by stress in women from the United States. Circulation 2005;111:472-9.

5. Sharkey SW, Windenburg DC, Lesser JR, et al. Natural history and expansive clinical profile of stress (tako-tsubo) cardiomyopathy. $J$ Am Coll Cardiol 2010;55:333-41.

6. Citro R, Rigo F, D'Andrea A, et al. Echocardiographic correlates of acute heart failure, cardiogenic shock, and in-hospital mortality in tako-tsubo cardiomyopathy. JACC Cardiovasc Imaging 2014;7:119-29.

7. Schneider B, Athanasiadis A, Schwab J, et al. Complications in the clinical course of tako-tsubo cardiomyopathy. Int J Cardiol 2014;176:199-205.

8. Isogai $\mathrm{T}$, Yasunaga $\mathrm{H}$, Matsui $\mathrm{H}$, et al. Out-of-hospital versus inhospital Takotsubo cardiomyopathy: analysis of 3719 patients in the Diagnosis Procedure Combination database in Japan. Int $J$ Cardiol 2014;176:413-7.

9. Brinjikji W, El-Sayed AM, Salka S. In-hospital mortality among patients with takotsubo cardiomyopathy: a study of the National Inpatient Sample 2008 to 2009. Am Heart J 2012;164:215-21.

10. Kwon SW, Kim BO, Kim MH, et al. Diverse left ventricular morphology and predictors of short-term outcome in patients with stress-induced cardiomyopathy. Int J Cardiol 2013;168:331-7.

11. Singh K, Carson K, Shah R, et al. Meta-analysis of clinical correlates of acute mortality in takotsubo cardiomyopathy. Am J Cardiol 2014;113:1420-8.

12. Parodi G, Bellandi B, Del Pace S, et al. Natural history of tako-tsubo cardiomyopathy. Chest 2011;139:887-92.

13. Elesber AA, Prasad A, Lennon RJ, et al. Four-year recurrence rate and prognosis of the apical ballooning syndrome. J Am Coll Cardiol 2007;50:448-52.

14. Looi JL, Wong CW, Khan A, et al. Clinical characteristics and outcome of apical ballooning syndrome in Auckland, New Zealand. Heart Lung Circ 2012;21:143-9.

15. Eitel I, von Knobelsdorff-Brenkenhoff F, Bernhardt P, et al. Clinical characteristics and cardiovascular magnetic resonance findings in stress (takotsubo) cardiomyopathy. JAMA 2011;306:277-86.

16. Chen HY, Gore JM, Lapane KL, et al. A 35-Year Perspective (1975 to 2009) into the Long-Term Prognosis and Hospital Management of Patients Discharged from the Hospital After a First Acute Myocardial Infarction. Am J Cardiol 2015;116:24-9.

17. Kostis WJ, Deng Y, Pantazopoulos JS, et al. Trends in mortality of acute myocardial infarction after discharge from the hospital. Circ Cardiovasc Qual Outcomes 2010;3:581-9.

18. Roger VL, Weston SA, Gerber Y, et al. Trends in incidence, severity, and outcome of hospitalized myocardial infarction. Circulation 2010;121:863-9.

19. El-Sayed AM, Brinjikji W, Salka S, Demographic SS. Demographic and co-morbid predictors of stress (takotsubo) cardiomyopathy. Am J Cardiol 2012;110:1368-72.

20. Zalewska-Adamiec M, Bachorzewska-Gajewska H, TomaszukKazberuk A, et al. Takotsubo cardiomyopathy: serious early complications and two-year mortality - a 101 case study. Neth Heart J 2016;24:511-9.

21. Redfors $B$, Vedad R, Angerås $\mathrm{O}$, et al. Mortality in takotsubo syndrome is similar to mortality in myocardial infarction - A report from the SWEDEHEART registry. Int J Cardiol 2015;185:282-9.

22. Tornvall P, Collste O, Ehrenborg E, et al. A Case-Control Study of Risk Markers and Mortality in Takotsubo Stress Cardiomyopathy. $J$ Am Coll Cardiol 2016;67:1931-6.

23. Stiermaier T, Moeller $\mathrm{C}$, Oehler $\mathrm{K}$, et al. Long-term excess mortality in takotsubo cardiomyopathy: predictors, causes and clinical consequences. Eur J Heart Fail 2016;18:650-6.

24. Ghadri JR, Wittstein IS, Prasad A, et al. International Expert Consensus Document on Takotsubo Syndrome (Part I): Clinical 
Characteristics, Diagnostic Criteria, and Pathophysiology. Eur Heart J 2018;39:2032-46.

25. Wells S, Riddell T, Kerr A, et al. Cohort Profile: The PREDICT Cardiovascular Disease Cohort in New Zealand Primary Care (PREDICT-CVD 19). Int J Epidemiol 2015.

26. Pylypchuk R, Wells S, Kerr A, et al. Cardiovascular disease risk prediction equations in 400000 primary care patients in New Zealand: a derivation and validation study. Lancet 2018;391:1897-907.

27. Deshmukh A, Kumar G, Pant S, et al. Prevalence of Takotsubo cardiomyopathy in the United States. Am Heart J 2012;164:66-71.
28. Akashi YJ, Goldstein DS, Barbaro G, et al. Takotsubo cardiomyopathy: a new form of acute, reversible heart failure. Circulation 2008;118:2754-62.

29. Grey C, Jackson R, Wells S, et al. Twenty-eight day and oneyear case fatality after hospitalisation with an acute coronary syndrome: a nationwide data linkage study. Aust N Z J Public Health 2014;38:216-20.

30. Wittstein IS, Thiemann DR, Lima JA, et al. Neurohumoral features of myocardial stunning due to sudden emotional stress. N Engl J Med 2005;352:539-48. 